\title{
Sizing up bacteria
}

Chances are you've never seen a bacterium with your naked eye. The largest physical dimension of a typical bacterium - the common rod-shaped E. coli, for example is on the order of $1 \mu \mathrm{m}$, about 100 times smaller than the width of a human hair. We've all seen bacteria, but only with the aid of a microscope.

You may wonder - why aren't some bacteria a lot bigger? Well, there's a fairly obvious answer: the constraints of molecular physics mean that a bacterium as big as a mouse, or even a flea, shouldn't work. As simple organisms lacking internal transport mechanisms, bacteria rely on random molecular diffusion to move necessary proteins and metabolites around within the cell. That's okay on a scale of $1 \mu \mathrm{m}$; not so good for anything much bigger.

After all, the basic mathematics of diffusion shows that the time required for a molecule to diffuse over a distance $D$ scales as $D^{2}$. A process taking about one-tenth of a second in a $1 \mu \mathrm{m} E$. coli would, in a cell 500 times as big, take 25,000 seconds, or about seven hours. Molecular biochemistry through diffusion would grind to a halt. Size comes with a cost. Bacteria have to remain small to keep all their interior cytoplasm close to the surface, for easy access to the external world.

However, there actually is a bacterium you can see with your naked eye; an unusual organism that has engineered a clever way around the physical limits of diffusion. The bacterium Epulopiscium can be as long as $600 \mu \mathrm{m}$ and one-fourth as wide; a few in your hand would look like some slender poppy seeds. It's an organism that, so far as biologists know, lives only in exotic realms - specifically, the guts of several species of tropical marine surgeonfish. Hence, the name Epulopiscium, which literally means "guest at the dinner of a fish".

How Epulopiscium manages to overcome the limits of diffusion is at once simple and profound. It illustrates how much biologists still have to learn about what a genome is and how it exerts influence over a living organism.

The key trick exploited by Epulopiscium is polyploidy - a vast parallel multiplication of its genomic machinery. Human cells are diploid - each cell contains two copies of the chromosomal DNA; one from each parent. Many bacteria are monoploid, having a single copy per cell, but biologists over the past decade or so have learned that

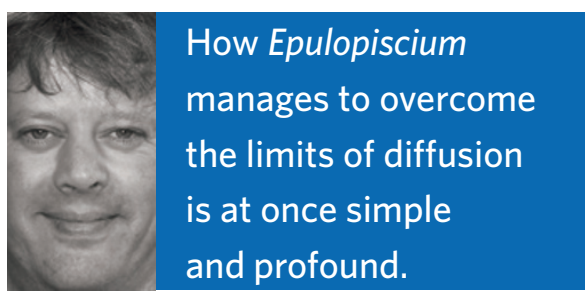

many others are not - many species of bacteria have hundreds of copies distributed throughout the cell. The multiplicity needn't stay fixed, but can vary during the bacterial lifetime. A freshwater bacterium known as Synechocystis PCC6803, for example, seems to have roughly 50 copies during some phases of its growth, and more than 200 during others.

Epulopiscium goes much further. A study from 2008 (J. Mendell et al., Proc. Natl Acad. Sci. USA 105, 6730-6734; 2008) found that the amount of DNA in a typical Epulopiscium cell is far larger than in a more normal bacterium. In principle, this could be because the genome of this bacterium is particularly large, but that turns out not to be the case. Epulopiscium has a fairly ordinary genome size (3.8 megabase) but has tens of thousands of distinct copies of this genome. They're located not just randomly throughout the bacterial cytoplasm, but strategically near to the cell membrane - close to the surface source of diffusing molecules.

Every one of these genomes is a small biomolecular factory. As a consequence, Epulopiscium has the capacity to transcribe any gene at many distinct locations within the cell, like a distributed parallel-processing system. Even with diffusion alone, distributed production means that proteins and metabolites get made near to where they will be used. Hence, Epulopiscium is a little like a microcolony, with different regions of the cell acting independently in response to local needs or stimuli, and therefore diffusion isn't a limitation on size.

In short, the genome-copying tricks allow this bacterium to be big without having a special mechanism for transport, as in more specialized organisms. What does Epulopiscium gain by being so large? That's not entirely clear, but researchers have some suspicions. As the bacteria feed within the host fish, they have to respond to digestive rhythms during the day, migrating long distances to different parts of the alimentary tract. Larger size seems to give cells greater control over location. Epulopiscium also faces potential predators in the form of various protozoans, and being large makes it impervious to all but the largest of these.

One more thing about this remarkable bacterium. Bacteria, as biological tradition has it, reproduce asexually, in sharp contrast with more complex multicellular organisms, many of which reproduce sexually. It's a simple division. Whereas single-celled bacteria simply pass their genetic material on to their offspring, multicellular organisms only pass on a fraction of their genetic material - the germline. The somatic DNA in all cells, with no role in reproduction, represents the cost of cellular diversification, which also goes along with bigger body size and metabolic capacity.

Epulopiscium seems to fall between these two extremes, as a kind of intermediate species. It reproduces in a peculiar way, forming multiple internal offspring before dividing into many parts. These huge bacterial cells manage to get some of the advantages of microbes with social lives, plus some extra benefits such as greater motility and higher resistance to predation.

The bacterium isn't what you (may have) thought it was. Biology textbooks may still introduce it to students as a monoploid creature reproducing by simple cell division, with two identical daughter cells carrying identical DNA, but the story is more sophisticated. Bacteria routinely exchange genetic material laterally, and genomes aren't the fixed structures we've been led to believe, but highly dynamic - changing and responding through a bacterium's life cycle.

And polyploidism, as exploited by Epulopiscium, is even among the simpler tricks (A. Oliverio and L. Katz, Genome Biol. Evol. 6, 482-488; 2014). You can break the chromosomes of another bacterium, Deinococcus radiodurans, into literally hundreds of pieces, and they'll spontaneously reassemble in only a few hours. Somehow, this genome has mechanisms allowing overlapping genome fragments to act as templates for repair, something that makes the bacterium extremely resistant to ionizing radiation.

Bacteria so big that you can see them may be surprising. But given that most bacteria on the planet have still not been well characterized, there are probably many more surprises yet in store.

\section{MARK BUCHANAN}

